\title{
An Improved Median Filtering Method and its Applications in Feature Extraction of GRIN Lens End Image
}

\author{
WU Song-lin,Zhang Bo andZhang Min
}

Faculty of Mechanical Engineering, XiJing University. Xi'an, the People's Republic of China710123

Keywords:GRIN lens end image processing, Feature extraction, Median Filtering.

Abstract.In this paper, animproved median filtering method for particular local grey level is presented andused to the image processing experiments of gradient-index (GRIN) lens end face. The principle and methodology of image processing for features extraction of defects in GRIN lens end are introduced, on the basis of the proposed median filtering, threshold segmentation, and morphological statistics of local featureinformation. In the method, the determination process of defects information is described abstractedly, including the pre-processing, classification and determination. Innovative experiment methods and promising results are presented in this paper.

\section{Introduction}

Recent years, a new type of optical lens, self-focus lens or GRIN lens ${ }^{[1]}$, has been developed, with its advanced functions such as imaging, collimating and focusing. It has been used broadly in optics during the past decades. To measure the production quality of the end face of GRIN lens is an important process in its production, and is generally based on the dimension and numerical measure of defects distributed on the face, such as scratches and smudge. It is generally completed on tool microscope or other optical instrument. There exist lower measuring precision and more manually operation in production testing of end face of GRIN lens.

With the development of technology, the application of GRIN lensis gradually increased, especially in the fiber optical connector. Scale production has the problems in measurement and control in production quality of GRIN lens emerged recently. Analysis system making use of computer image processing to detect quality of the end face quality of fiber optic and other optical lens has been reported recently[2][3]. However, they rarely refer to the applications in the end face measurement of GRIN lens. There are some particular characteristicsin the image of GRIN lens end, because of the different incline areas on the two end face. Some adjustments and alternationsmust be made to suit the characteristicswhen traditional image processing methods are used. A new median filtering for particular local grey level is put forward and used to manage this kind of images in this paper. To accomplish the defects feature extraction through image processing of GRIN lens end, a series of methods are also presented, including the pre-processing, classification and features determination based on the morphology stochastic. As such, the experiment instrument and processing results are presented to show the efficient.

\section{The Improved Median Filtering Method}

The median filtering is a nonlinear data processing method to decrease the noise, proposed by Turkey in 1971. Its fundamental principle is replace a data point in digital image or digital series by a median value calculated according to the every value in its neighborhood. The method is to remove any isolated noise point and make the surrounding pixels approach the true value in a great 
extent. A moving mode with some structure is generally used in its way. The pixels in the mode are sorted according to their sizes to produce a monotonically rising or decreasing two-dimensional data series. A group of data $x_{1}, x_{2}, \ldots, x_{n}$ is sequenced as $x_{n} \leq x_{i 2} \leq x_{i 3} \leq \cdots \leq x_{i m}$. Then the median value then is defined as following

$$
\mathrm{y}=\operatorname{med}\left\{x_{1}, x_{2}, \ldots, x_{n}\right\}=\left\{\begin{array}{cl}
x_{i \frac{n+1}{2}} & \text { if } n \text { is odd } \\
\frac{1}{2}\left[x_{i \frac{n+1}{2}}+x_{i\left(\frac{n}{2}+1\right)}\right] & \text { if } n \text { is even }
\end{array}\right.
$$

Suppose the input series $\left\{x_{i}, i \in N\right\}, \mathrm{N}$ is a set of natural number or a subset. Its window length is $\mathrm{n}$. Then the filtering output is

Where $i \in N: u=(n-1) / 2$.

$$
y_{i}=\operatorname{med}\left\{x_{i}\right\}=\operatorname{med}\left\{x_{i-u}, \ldots, x_{i}, \ldots, x_{i+u}\right\}(2)
$$

If $\left\{x_{i j},(i, j) \in N^{2}\right\}$ are the grey levels in a digital image, the two-dimension filtering with A, the filtering window, can be defined as

$$
y_{i j}=\operatorname{med}_{A}\left\{x_{i j}\right\}=\operatorname{med}\left\{x_{i+r, j+s},(r, s) \in A(i, j) \in N^{2}\right\}(3)
$$

The two-dimension filtering is

$$
\mathrm{g}(\mathrm{x}, \mathrm{y})=\operatorname{med}\{f(x-k, y-l),(k, l) \in A\}(4)
$$

The function, $\mathrm{f}(\mathrm{x}, \mathrm{y})$ and $g(x, y)$, is the original and processed image respectively. $\mathrm{A}$, the mode, is generally a $3 \times 3$, or $5 \times 5$ pixel region.

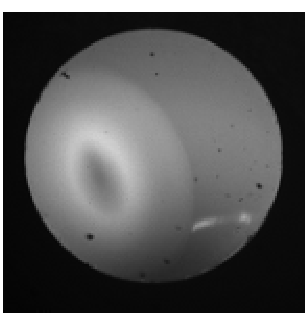

(a)

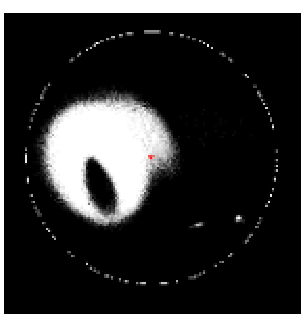

(b)

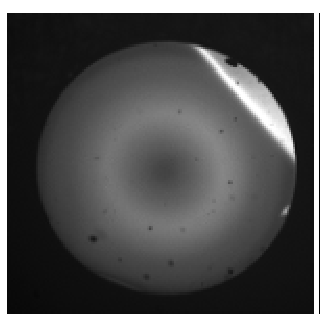

(c)

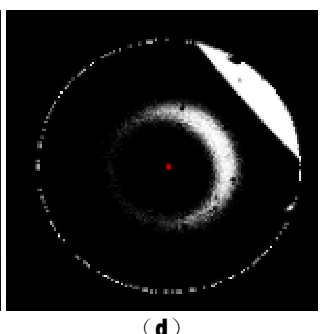

(d)

Fig.1 the original images and their properties of GRIN lens end

The image of typical GRIN lens end, however, show clearly different regulations of grey level distribution. As shown in figure 1, (a) and (c) is the one end image and another oneof a GRINlens respectively. (b) and (d) 'express their distribution characteristics of over enough bright grey level in the images. To suit to these characteristics in images processing and weak their influence to the following feature extraction, the median filtering method discussed above is improved. A, the mode of pixel region, is designed as a $3 \times 3$ pixel area positioned right on the top left corner of the point to be processed. The median value then is defined on the basis of all 8 pixel values, same as function (3), excepting the point in the area to be processed. In this way, the impact from the relatively brighter grey level is decreased to a great extent. 


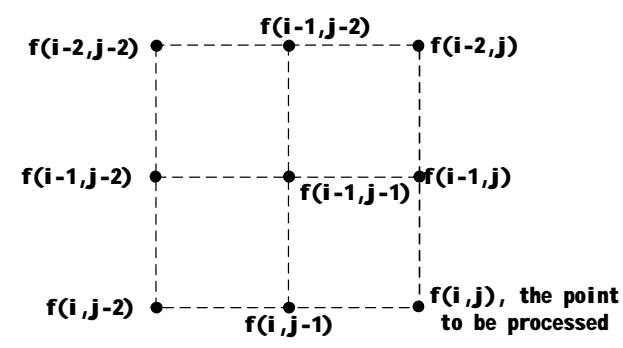

Fig. 2 the mode designed

In this case,output of the improved median filtering isdefined as function (5). Where, $w(i, j)$ means the weightings calculated according to distance from the point to be processed. The weighting is increased with the distance.

$$
\mathrm{g}(\mathrm{x}, \mathrm{y})=\operatorname{med}\{w(i, j) f(x-k, y-l),(k, l) \in A, \text { except } f(i, j)\}(5)
$$

\section{Image Processing Experiments}

To accomplish the image processing for defects feature extraction, a measurement installation and processing system have been designed as shown in Fig 3 and Fig 4. The measurement installation includes light source, CCD camera and condensers. The end face of GRIN lens fixed on a platform is lighted by a collimated beam produced by the light source, through the condenser aperture and condenser lens. A CCD camera is used to receive the image in a proper focal length, which transfers the image to the computer of image processing system. In case the satisfactory image is obtained, it is transferred to image processing system.

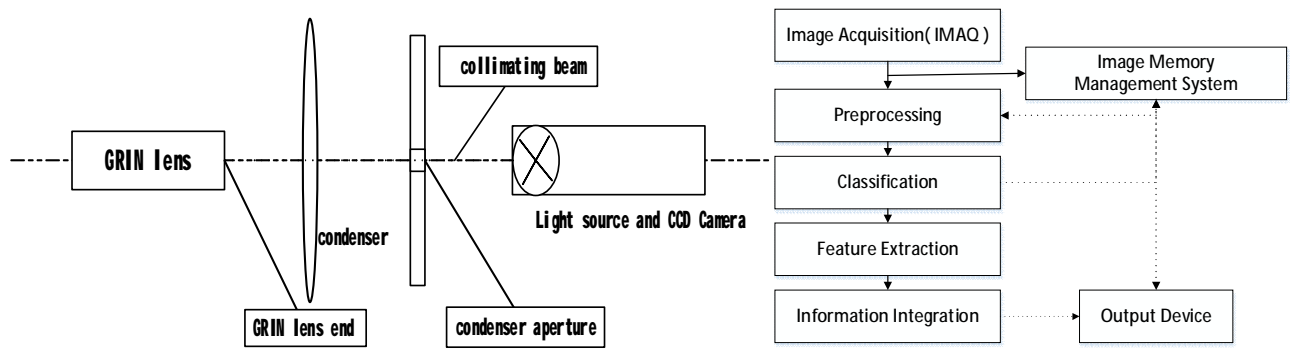

Figure 3 Measurementinstallation of GRIN lens end

Figure 4 SystemComposition

The image processing system includes image acquisition and memory management, pre-processing, classification, feature extraction and information integration. The final result is exported through output device.

Typical feature extraction process is shown in the figure 5, in which (1), (5) and (9) are three kind of images with scratch, irregularfault area and chippings respectively. The images with label (2), (6) and (10) are the output images through the improved median filtering. It can be clearly seen that the information on features are enhanced and the affection of relative brighter grey level is weaken.

On the basis of the application of the median filtering, outline and form characteristics of the defects features on the images can be extracted easily through traditional image method. As shown in figure 5 (3), (4), (7), (8), (11) and (12), the outermost outline and the form of defects features are determined and labeled. Take (7) and (8) for example, the biggest defect area is $170.55\left(\mu \mathrm{m}^{2}\right)$ and the coordinate of its form center are $1.21 \mathrm{~mm}$ and $1.97 \mathrm{~mm}$ through calculations. The origin of coordinate is on the top left corner of the image, as shown in Fig.5. 


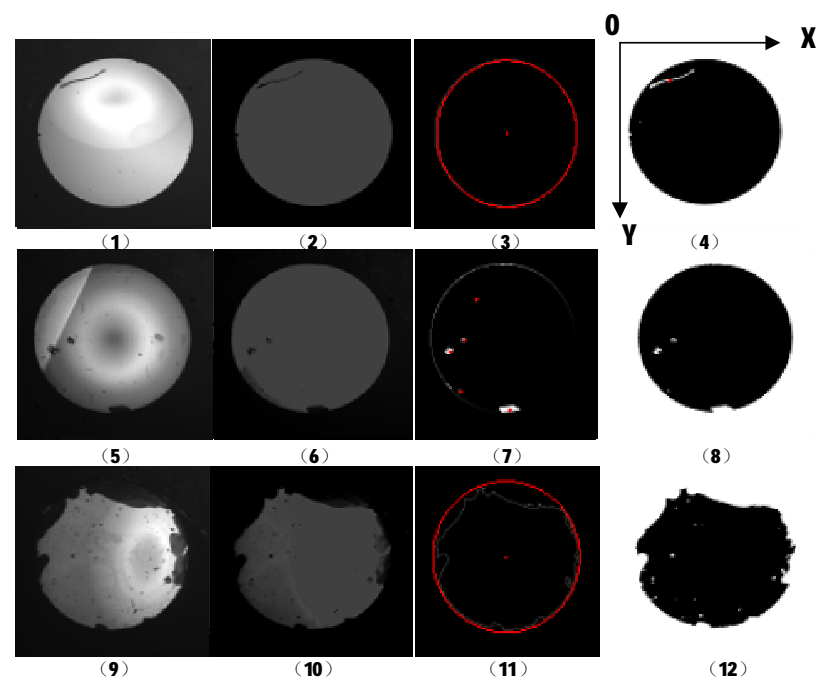

Fig.5 the defects features determination of GRIN lens end

\section{Conclusions}

Through detailed analysis of the characteristics of GRIN lens end image, animproved median filtering method is developed and applied to determination of the defects features in these images. The related experiment installationhas been designed and pre-processing algorithm and classification procedure have been presented for experimental purpose. Promising experiment results show that the presented methods are effective.

\section{Acknowledgement}

This work is financially supported by the Scientific Research Fund Project of Xijing University "Synthesis analysis of micro structural characteristics of engineering surface and its applications".

\section{Reference}

[1]HaruikoTsuehiy a, Appl. Opt., Vol.16, P1328-2331 (977).

[2]Mao Y.X. et al., Gradient-index fiber lens proposed for ultra-small probes used in biomedical imaging [J]. Appl. Opt., 2007, 46(23):5887-5894

[3]Jose P.C.Palais, Appl. Opt., Vol.19, p2011-2018(1980).

[4]Wang C, Mao Y X, et al., Numerical analysis of GRIN lens based miniature probes for optical coherence tomography [J]. Optics and Precision Engineering, 2011, 19(9):2300-2307.

[5]L H, Liu A. Tong $\mathbf{J}$ et al., Nondestructive measurement of gradient refractive index rod lens [J].Review of Scientific Instruments, 2010, 81(10):103-104.

[6]Y CH, Kim D Y., A simple reflection type two dimensional refractive index profile measurement technique for optical waveguides[J]. Optics Communications, 2006, 262(2):206-210.

[7]Sun X H, Ma H, et al., The measurement of refractive index profile and aberration of radial gradient index lens by using imaging method [J]. Optics and Laser Technology, 2004, 36(2):163-166. 